\title{
Decision Priming in an Air-to-Ground Attack Decision Scenario
}

\author{
Marc Winterbottom \\ Air Force Research Laboratory \\ marc.winterbottom@mesa.afmc.af.mil
}

\author{
Byron Pierce \\ Air Force Research Laboratory \\ byron.pierce@mesa.afmc.af.mil
}

\author{
Robert Patterson \\ Washington State University \\ rpatter@wsu.edu \\ Logan Williams \\ Air Force Research Laboratory \\ logan.williams@mesa.afmc.af.mil
}

\author{
Lisa Fournier \\ Washington State University \\ lfournier@wsu.edu \\ Ryan Amann \\ Link Simulation and Training \\ ryan.amann@mesa.afmc.af.mil
}

\begin{abstract}
Motivation - Validate a System Dynamics model of decision speed for a scenario relevant to an Air Force mission task. Research approach - Extend a decision priming basic research paradigm to a more applied case. Findings/Design - Decision priming was found to occur with more complex stimuli relevant to an air-to-ground target identification scenario. A system dynamics model was developed to predict those results. Research limitations/Implications - While decision delays of approximately 100 milliseconds were found here, it remains to be determined whether decision priming extends to decision processes extending over seconds or minutes. Originality/Value - A temporally-based method of modeling a decision process has been validated for an applied air-to-ground target identification scenario. Many other decision models lack this time component and are therefore of limited use in time-critical situations. Take away message - Tactical situations where information is processed rapidly and sequentially can result in decision priming.
\end{abstract}

Keywords

Decision priming, system dynamics modeling, decision modeling

\section{INTRODUCTION}

Robust decision making plays a crucial role in many real-world situations, especially in complex operational environments such as those encountered by Air Force personnel during mission execution. Often, these decisions are made under extreme time constraints, thus it is critically important to understand how the time course of information may affect the speed of decision making. In the present study, we investigated reaction time for a simulated groundtarget identification scenario that an Air Force pilot might encounter as part of a ground attack mission, a situation in which rapid and accurate decision making is critical, and where information regarding the target may be transmitted to the pilot at different times under high workload conditions. We employed a task that required participants to decide whether a ground vehicle matched a target description, which was defined by a conjunction of two cues, and thus whether to complete or abort the mission.

We predicted that faster discrimination of one cue would lead to decision priming. Such priming should occur when initial information generates the expectation that a given decision is appropriate, and that expectation speeds up or slows down decision making. Specifically, we predicted that decisions to engage the target would be faster when both target cues matched expectations, and decisions to abort the mission would be slower when the two cues conflicted. The basis of the present study was a series of studies in the basic literature in which reaction time in identifying the conjunction of simple features (e.g. orientation, color, shape, size, spatial frequency, etc), was measured, and decision priming was shown to occur (Fournier et al., 1998, 2000, 2007). These authors found that a more rapidly processed cue (e.g. color) could prime (speed up) target identification that involved a conjunction of two cues (e.g. color and also shape). However, when color matched expectations and shape did not, decision time was slowed relative to when neither cue matched the target description. Here, we are extending this basic research paradigm to a more complex scene involving an air-to-ground target identification scenario, while maintaining similar independent variables.

We also attempted to predict the results of both the earlier basic research study, as well as the results of the present study using a System Dynamics model. System dynamics modeling involves analyzing the dynamics of complex systems by casting them as systems of interconnected positive and negative feedback loops (Forrester, 1968). System dynamics is a temporally-based modeling approach. Thus, it may be well-suited for use in tactical decision scenarios where time is critical, and may offer an advantage over other modeling approaches that do not incorporate a time component. 
Winterbottom $\bullet$ Patterson $\bullet$ Fournier $・$ Pierce $・$ Williams $\bullet$ Amann

Decision Priming in an Air-to-Ground Attack Decision Scenario

\section{METHOD}

Participants. 12 volunteers with normal or corrected to normal vision. Stimuli and apparatus. American M1A2 and Russian T-62 tank models with either desert (brown) or forest camouflage (green) were presented against a desert terrain background. A Samsung rear-projection HDTV was used to present the stimuli. MetaVR VRSG 5.0 was used to generate the terrain and 3D models. A PC-based threat generator was used to control the experiment and record observer responses. Procedure. The experiment was divided into three blocks of trials. In one block, participants responded only to the color of the target. In a second block, participants responded only to the vehicle type (i.e. shape, or M1A2 vs. T-62). In a third block, participants responded to the conjunction of the two cues. Text displayed on the screen informed the participant prior to each block as to which cue to respond. Prior to each trial, the target was identified (e.g. Brown Russian). The target was then displayed for $100 \mathrm{msec}$ and the participant responded, using a joystick, as to whether the vehicle was the target (yes/no). Accuracy and reaction time were recorded.

\section{RESULTS}

The various cue combinations resulted in different reaction times. For target present decisions mean reaction times were 708, 786, and $771 \mathrm{msec}$ for Color only, Vehicle Type only, and both Color and Vehicle Type, respectively. For target absent decisions mean reaction times were 817, 703, and $713 \mathrm{msec}$ for Color but not Vehicle Type, neither Color nor Vehicle Type, and Vehicle Type but not Color, respectively.

Based on the prior priming literature, only selected comparisons were of interest: 1) mean reaction time based on Color alone was significantly faster than that for Vehicle Type alone $[t=-3.36, p<0.01]$; 2) the combination of correct Color but incorrect Vehicle Type (cue conflict) resulted in slower reaction times $[t=6.1, p<0.001]$ compared to another target absent condition (neither Color nor Vehicle Type matched target description); and 3) the combination of correct Color and Vehicle Type was not significantly different than for vehicle type alone $(t=-0.77, p=0.23)$. Thus decision speed was slowed when the more rapidly processed cue signaled a "target present" response that then had to be countermanded when the more slowly processed cue did not match the target description. Additionally, processing time for the "Target Present" combination of Color and Vehicle Type cues under these conditions did not require any longer than for the Vehicle Type cue alone.

\section{System dynamics model}

The System Dynamics model was composed of three levels of processing: (1) feature integration mechanisms, (e.g. "M1A2", "T-62", "brown", and "green"); (2) individual decisions made about individual features, which involves decision evidence integration processes ("brown decision evidence", "green decision evidence", "M1A2 decision evidence", and "T-62 decision evidence", followed by "hue comparison" and "shape comparison" mechanisms); and (3) a central decision process rendering a "target present" or "target absent" decision. An analogous model was created to predict the results of the earlier basic research experiment using simpler cues with differing time constants. The simulated results of each model resulted in decision priming similar to that found empirically.

\section{CONCLUSION}

Decision priming was shown to occur for more complex stimuli relevant to a simplified air-to-ground target identification scenario. Moreover, these results were accurately predicted by a system dynamics model of decision priming. An important aspect of this model is the simulation of decision competition, which leads to decision priming. Decision priming is caused by early initial activation of a central decision process which must be overridden, thereby slowing the final decision process. In real-world operational situations where multiple stimuli are present, attention may be distracted, difficult sensory-motor tasks must be performed (e.g., flying an aircraft), and rapid reaction to threats is critical, the increase in reaction time (priming) due to decision competition could be a significant factor. By exploring the operation of the system dynamics model under different simulated conditions, the time course of decision priming can be examined, and methods of reducing or eliminating delays in decision making may be discovered.

\section{ACKNOWLEDGMENTS}

This work was supported by USAF Contract FA8650-05-D-6502 to Link Simulation and Training. We thank Lt Adam Pohl for his excellent technical support on this project.

\section{REFERENCES}

Forrester, J.W. (1968). Principles of systems. Waltham, MA: Pegasus Communications.

Fournier, L. R., \& Eriksen, C. W., \& Bowd, C. (1998). Multiple feature discrimination faster than single feature discrimination within the same object? Perception \& Psychophysics, 60, pp. 1384-1405.

Fournier, L. R., Bowd, C., \& Herbert, R. J. (2000a). Interference from multi-dimensional objects during feature and conjunction discriminations. Quarterly Journal of Experimental Psychology, 53A, pp. 191-209.

Fournier, L., Patterson, R., Dyre, B., Weidiger, M., \& Winters, R. (2007). Conjunction benefits and costs reveal decision priming for first-order and second-order features. Perception \& Psychophysics, 69, pp. 1409-1421. 